\title{
Contributions of Inelastically Scattered Electrons to Defect Images.
}

\author{
M. A. Kirk*, R. Twesten**, S. P. Martin***, C. J. D. Hetherington*** and M. L. Jenkins*** \\ *Materials Science Division, Argonne National Laboratory, Argonne, IL 60439 \\ **Seitz Materials Research Laboratory, University of Illinois, Urbana-Champaign \\ ***Department of Materials, University of Oxford, Oxford, UK
}

We have made measurements of the contribution of inelastically scattered electrons to images of dislocations in $\mathrm{Ni}_{3} \mathrm{Ga}$ and nanometer-sized defects in ion-irradiated $\mathrm{Au}$ under weak-beam darkfield diffraction conditions [1]. The purpose is to determine the conditions for data acquisition required to eventually make detailed and quantitative comparisons to simulations of images for various defect models, thus determining defect structure, composition, and local strain field. Such image simulations usually consider only elastically scattered electrons, and thus it is important to understand and possibly eliminate the contribution of inelasically scattered electrons to the experimental images for quantitative comparisons with image simulations.

Experimental data have been acquired with either JEOL 2010F or 3000F microscopes, both equipped with Gatan Imaging Filter electron spectrometers. Samples examined in the 2010F were $\mathrm{Au}$, ion-irradiated to low dose $\left(10^{11} \mathrm{Kr}\right.$ ions at $1 \mathrm{MeV}$ energy) to form individual defects (1$10 \mathrm{~nm}$ sized Frank dislocation loops and partial stacking fault tetrahedra). Samples examined in the $3000 \mathrm{~F}$ were $\mathrm{Ni}_{3} \mathrm{Ga}$ with long dislocation defects. Imaging conditions included weak-beam dark-field with deviation parameter generally $>0.2 \mathrm{~nm}^{-1}$. Energy filter slit width was set to 10 $\mathrm{eV}$ and centered on the zero loss peak in both instruments to obtain images produced by elastically scattered electrons. In the Au experiment an energy window was also set to image the $10-40 \mathrm{eV}$ loss range to investigate the contribution to the defect images of plasmon-loss electrons. Electron intensity in defect images and backgrounds was measured by summing pixel values in appropriately sized rectangles using Digital Micrograph (Gatan) software.

Examples of defect images in the exact same area in Au for three imaging conditions, unfiltered, zero-loss, and plasmon-loss, are illustrated in Fig. 1. Note the great similarity in image resolution and contrast for the first two, and some loss of resolution (at four times the exposure time of the first two) for the image formed by plasmon-loss electrons only. Integrated intensity measurements on four of the defects and neighboring backgrounds showed between 15 and $20 \%$ of the total contrast to come from inelastically scattered electrons, and about one third of that could be assigned to plasmon-loss electrons. Making the assumption that the electron thermal diffuse scattering does not contribute to image contrast [2], this means that about two thirds of the inelastic scattering that is image forming is due to single electron events [3]. A possible example of such scattering is found in weak defect images which are formed at 1.3 times the 200 Bragg angle, corresponding to M-shell ionization-loss electrons in $\mathrm{Au}(2.2 \mathrm{keV})$. These very weak images cannot be found in zero-loss electrons at this position in reciprocal space.

An example of a much stronger contribution to defect (dislocation) images in $\mathrm{Ni}_{3} \mathrm{Ga}$ for inelastically scattered electrons is illustrated in Fig. 2. Here it was found that such electrons constitute $50-60 \%$ of the image in thin area, and as much as $90 \%$ in thicker area. In this lighter 
material advantages to energy filtering on zero loss electrons are indeed great, enhancing both image peak-to-background contrast and image resolution, especially in thicker area [4].

[1] Characterization of Radiation Damage by Transmission Electron Microscopy, M. L. Enkins and M. A. Kirk, Inst. Phys. Publ., Bristol, UK, 2001, pp. 1-224.

[2] S. L. Cundy, A. Howie and U. Valdre', Phil. Mag. 20 (1969) 147.

[3] C. J. Humphreys and M. J. Whelan, Phil. Mag. 20 (1969) 165.

[4] This research is supported by the U.S. Department of Energy, Office of Science, and the Department of Materials, University of Oxford, UK.
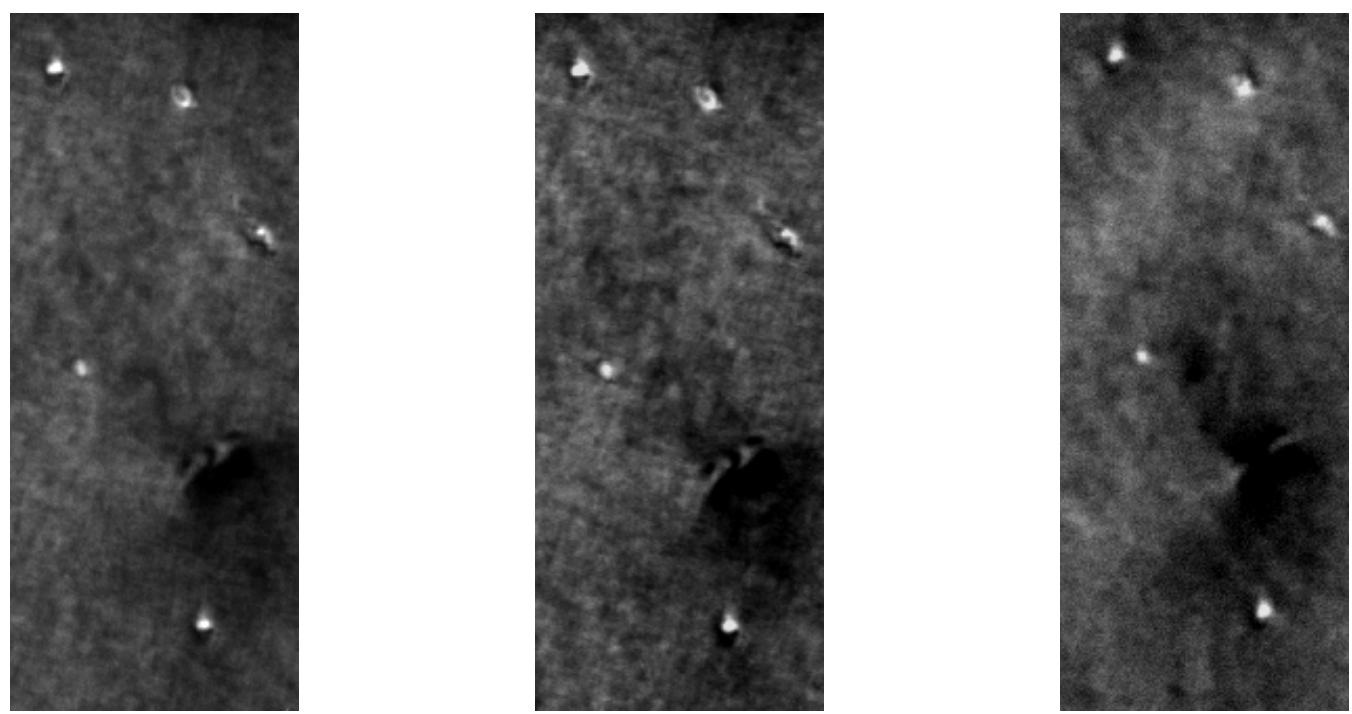

Fig. 1. Defect images in ion-irradiated Au. Left: unfiltered, middle: filtered on zero-loss peak with $10 \mathrm{eV}$ slit width, right: filtered on plasmon-loss electrons with $10-40 \mathrm{eV}$ window.
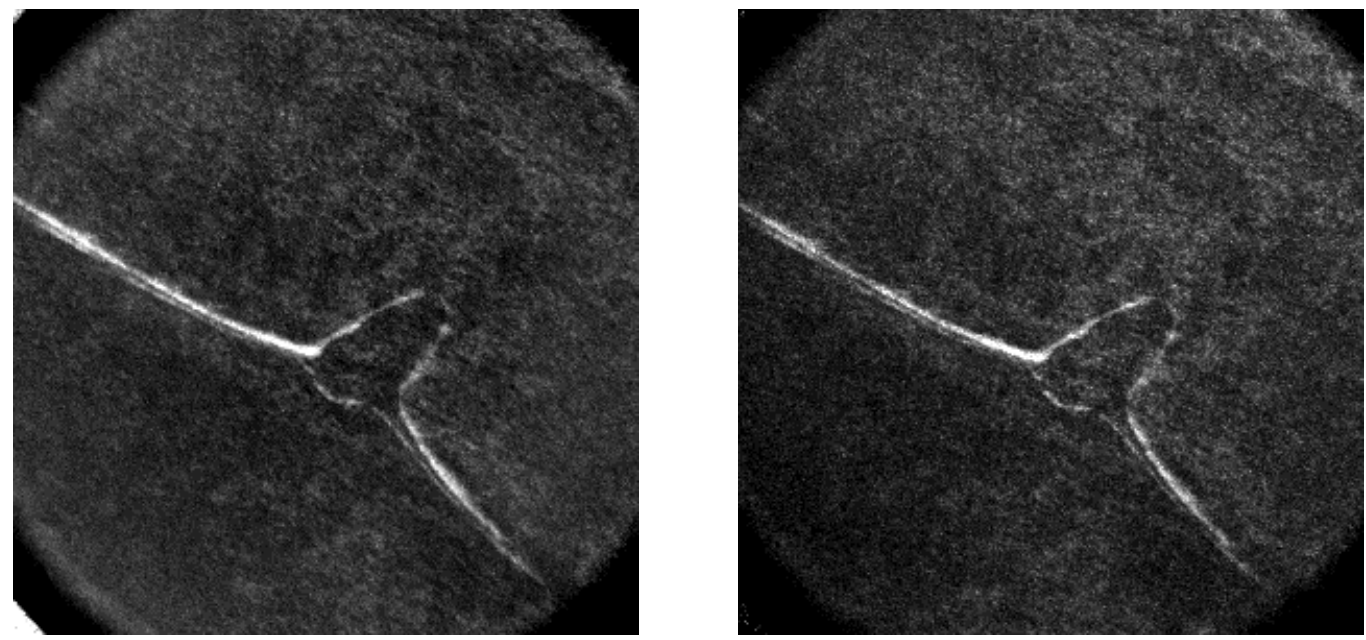

Fig. 2. Dislocation images in $\mathrm{Ni}_{3} \mathrm{Ga}$ : left image unfiltered and right image filtered on zero-loss peak with $10 \mathrm{eV}$ slit width. 